\title{
An Investment and Consumption Problem with CIR Interest Rate and Stochastic Volatility
}

\author{
Hao Chang' and Xi-min Rong ${ }^{2}$ \\ ${ }^{1}$ Department of Mathematics, Tianjin Polytechnic University, Binshui West Road 399, 300387 Tianjin, China \\ ${ }^{2}$ School of Science, Tianjin University, Wei-jin Road 72, 300072 Tianjin, China \\ Correspondence should be addressed to Hao Chang; ch8683897@126.com
}

Received 6 March 2013; Accepted 13 May 2013

Academic Editor: Ryan Loxton

Copyright (c) $2013 \mathrm{H}$. Chang and X.-m. Rong. This is an open access article distributed under the Creative Commons Attribution License, which permits unrestricted use, distribution, and reproduction in any medium, provided the original work is properly cited.

\begin{abstract}
We are concerned with an investment and consumption problem with stochastic interest rate and stochastic volatility, in which interest rate dynamic is described by the Cox-Ingersoll-Ross (CIR) model and the volatility of the stock is driven by Heston's stochastic volatility model. We apply stochastic optimal control theory to obtain the Hamilton-Jacobi-Bellman (HJB) equation for the value function and choose power utility and logarithm utility for our analysis. By using separate variable approach and variable change technique, we obtain the closed-form expressions of the optimal investment and consumption strategy. A numerical example is given to illustrate our results and to analyze the effect of market parameters on the optimal investment and consumption strategies.
\end{abstract}

\section{Introduction}

The first treatment of the investment and consumption problem in a continuous-time framework originated in the seminal papers of Merton [1,2]. Merton used dynamic programming principle to construct an explicit optimal portfolio for power and logarithm utility. Later on, this problem attracted more and more attention and inspired literally hundreds of extensions and applications. For example, Fleming and Zariphopoulou [3] and Vila and Zariphopoulou [4] studied optimal consumption and portfolio decisions with borrowing constraints to maximize the total expected discounted utility coming from consumption; Duffie et al. [5] investigated the optimal investment and consumption strategy in incomplete markets; Dumas and Luciano [6], Shreve and Soner [7], Liu and Loewenstein [8], and Dai et al. [9] focused on the investment and consumption problem with transaction costs under different market assumptions. But these models were investigated under the assumption that risk-free interest rate and the volatility of the stock were supposed to be constants.

However, two aspects are worthy to be further explored based on the above-mentioned literature. On the one hand, risk-free interest rate in most of the above-mentioned literature is assumed to be constant, which is contrary to the fact. Interest rate is not always fixed in our real life. There is much literature documenting the term structure of interest rate, such as Ho and Lee [10], Vasicek [11], and Cox et al. [12]. Therefore, many investors find that the optimal portfolios with stochastic interest rates are more practical. In recent years, many scholars have studied the portfolio selection problem under stochastic interest rate models. Korn and Kraft [13] and Grasselli [14] considered the optimal portfolios under Ho-Lee model and Vasicek model and CIR interest rate dynamics, respectively, and obtained the explicit solutions under the expected utility criterion. Deelstra et al. [15], Josa-Fombellida and Rincón-Zapatero [16], and Gao [17] investigated the investment strategies for pension funds with stochastic interest rate in different situations. Deelstra et al. [18] analyzed the optimal investment problem in a CIR framework and derived the closed-form solution of the optimal investment and consumption strategy by assuming the completeness of financial markets. Fleming and Pang [19] applied stochastic optimal control theory to an investment and consumption problem with stochastic interest rate and wished to maximize the total expected discounted utility of 
consumption but have no ways to obtain the explicit expressions and only prove the existence of solution by using subsupersolution method. Munk and Sørensen [20] characterized the solution to the investment and consumption problem with stochastic changes in the opportunity set and suggested that the hedge portfolio is more sensitive to the form of the term structure than to the dynamics of interest rate.

On the other hand, there is more and more attention paid to the portfolio selection problem with stochastic volatility (SV). Stochastic volatility has been recognized as an important factor of stock dynamics and can demonstrate many well-known empirical features, such as volatility smile and volatility clustering. Heston's SV model [21] is the simplest but important one. Kraft [22] studied the optimal portfolio with stochastic volatility and obtained the closed-form solution. Fleming and Hernández-Hernández [23] and Chacko and Viceira [24] analyzed an investment and consumption problem with SV in different market models, respectively. Li et al. [25] considered SV model in the insurance market and obtained the closed-form solution to the optimal investment and insurance strategies in a mean-variance framework. Sasha and Zariphopoulou [26], Castañeda-leyva and Hernández-Hernández [27], and Liu [28] applied different methods to investigate an investment and consumption model in different stochastic environments, where interest rate and return rate and volatility are supposed to be stochastic. Michelbrink and Le [29] proposed a martingale approach to tackle a consumption model with jump diffusions and obtained the optimal investment and consumption strategies. $\mathrm{Li}$ and $\mathrm{Wu}[30$ ] assumed that risk-free interest rate is driven by the CIR model and the volatility of the stock is described by Heston's SV model and obtained the optimal investment strategy to maximize the power utility of terminal wealth. Noh and Kim [31] investigated an investment and consumption model with stochastic interest rate and stochastic volatility, where interest rate model and the volatility model are correlated with the stock price. Noh and Kim analyzed the existence of the optimal portfolio, but did not obtain the closed-form solution. This paper considers one optimal consumption problem based on Li and Wu's model and uses the same approach as Liu [28] to solve corresponding nonlinear partial different equation. In addition, we obtain the explicit expressions of the optimal investment and consumption strategy in the power and logarithm utility cases.

As far as we know, there is little work in the literature on the investment and consumption problem with stochastic interest rate and stochastic volatility, and only Noh and Kim [31] studied this problem so far and choose the total expected discounted utility coming from consumption as the objective function. Therefore, in this paper, we assume that risk-free interest rate is driven by the CIR model and the volatility of stock is described by Heston's stochastic volatility model. Our objective is to maximize the expected discounted utility of consumption and terminal wealth. Firstly, we use dynamic programming principle to obtain the HJB equation for the value function and choose power utility and logarithm utility as our analysis. Secondly, by applying separate variable approach and variable change technique, we obtain the closedform solutions to the optimal investment and consumption strategy. A numerical example is given to illustrate the results obtained and analyze the impact of market parameters on the optimal investment and consumption strategy. This paper has three main contributions: (i) the optimal investment and consumption strategies under stochastic interest rate model and stochastic volatility model are studied, which is the more important extension of the work of $\mathrm{Li}$ and $\mathrm{Wu}$ [30]; (ii) the expected discounted utility of consumption and terminal wealth is chosen as the objective function, which is different from Noh and Kim [31], and our objective function is more practical; (iii) we use the same approach as Liu [28] to solve the nonlinear second-order partial different equation such that the closed-form solutions are obtained, which is the most important innovation of this paper.

The remainder of this paper is organized as follows. The financial market and wealth process and optimization criterion are presented in Section 2. In Section 3, we use dynamic programming principle to derive the HJB equation for the value function and obtain the closed-form solutions to the optimal investment and consumption strategy in the power and logarithm cases. In Section 4, we propose numerical illustration and sensitivity analysis. Section 5 concludes the paper.

\section{Problem Formulation}

Assume that there are no transaction costs or taxes in the financial market and all assets can be traded continuously. $\left(W_{1}(t), W_{2}(t)\right)$ is a two-dimensional well-defined and independent adapted Brownian motion in a given filtered complete probability space $\left(\Omega, \mathscr{F},\left\{\mathscr{F}_{t}\right\}_{t \in[0, T]}, P\right)$, where $T$ is a positive constant representing fixed and finite investment horizon; $\left\{\mathscr{F}_{t}\right\}_{t \in[0, T]}$ is an information filtration generated by $\left(W_{1}(t), W_{2}(t)\right)$.

2.1. The Financial Market. Assume that the financial market is composed of two assets: one risk-free asset (e.g., a bank account) and one risky asset (e.g., a stock). The price process of the risk-free asset at time $t$ is denoted by $B(t)$, which satisfies

$$
d B(t)=r(t) B(t) d t
$$

where $r(t)$ is the stochastic process and is supposed to be driven by the CIR model

$$
\begin{array}{r}
d r(t)=(\theta-c r(t)) d t+\sigma_{0} \sqrt{r(t)} d W_{1}(t), \\
r(0)=r_{0}>0,
\end{array}
$$

where $\theta, c$, and $\sigma_{0}$ are positive constants satisfying $2 \theta>\sigma_{0}^{2}$. It is well known that $r(t)>0$ for all $t \geq 0$ (see Cox et al. [12]).

The price process $S(t)$ of the risky asset is as follows:

$$
\begin{array}{r}
d S(t)=S(t)\left[(r(t)+k \eta(t)) d t+\sigma_{1} \sqrt{\eta(t)} d W_{2}(t)\right], \\
S(0)=s_{0}>0,
\end{array}
$$


where $k$ and $\sigma_{1}$ are positive constants, and $\eta(t)$ is driven by another CIR model

$$
\begin{array}{r}
d \eta(t)=(b-a \eta(t)) d t+\sigma_{2} \sqrt{\eta(t)} d W_{2}(t), \\
\eta(0)=\eta_{0}>0,
\end{array}
$$

where $b, a$, and $\sigma_{2}$ are positive constants satisfying $2 b>\sigma_{2}^{2}$. We have also $\eta(t)>0$ for all $t \geq 0$.

2.2. The Wealth Process. During the time horizon $[0, T]$, we suppose that the initial wealth of an investor is denoted by $x_{0}>0$, and the wealth process at time $t$ is denoted by $X(t)$. A trading strategy is a pair of stochastic processes denoted by $(\pi(t), C(t))$, where $\pi(t)$ is the amount invested in the stock, and $C(t)$ is the consumption rate. The amount invested in the risk-free asset is $X(t)-\pi(t)$. Therefore, wealth process evolves according to the following stochastic differential equation:

$$
\begin{array}{r}
d X(t)=(X(t)-\pi(t)) \frac{d B(t)}{B(t)}+\pi_{t} \frac{d S(t)}{S(t)}-C(t) d t, \\
X(0)=x_{0}>0 .
\end{array}
$$

Considering (1) and (3), we get

$$
\begin{aligned}
d X(t)= & (r(t) X(t)+\pi(t) k \eta(t)-C(t)) d t \\
& +\pi(t) \sigma_{1} \sqrt{\eta(t)} d W_{2}(t), \quad X(0)=x_{0}>0 .
\end{aligned}
$$

\subsection{The Optimization Criterion}

Definition 1 (admissible strategy). An investment and consumption strategy $(\pi(t), C(t))$ is said to be admissible if the following conditions are satisfied.

(i) $(\pi(t), C(t))$ is progressively measurable.

(ii) $E\left[\int_{0}^{T}\left(\pi(t) \sigma_{1} \sqrt{\eta(t)}\right)^{2} d t\right]<\infty$.

(iii) For all initial conditions $\left(t_{0}, r_{0}, \eta_{0}, x_{0}\right) \in[0, T] \times$ $(0, \infty)^{3}$, the wealth process $X(t)$ with $X(0)=x_{0}>0$ has a pathwise unique solution.

Assume that the set of all admissible strategies is denoted by $\Gamma=\{(\pi(t), C(t)): t \in[0, T]\}$. The investor wishes to maximize the expected discounted utility of the intermediate consumption and terminal wealth. Mathematically, the objective function is expressed as

$$
\begin{aligned}
\underset{(\pi(t), C(t)) \in \Gamma}{\operatorname{Maximize}} E[ & \alpha \int_{0}^{T} e^{-\beta t} U_{1}(C(t)) d t \\
& \left.+(1-\alpha) e^{-\beta T} U_{2}(X(T))\right],
\end{aligned}
$$

where $U_{1}(\cdot)$ and $U_{2}(\cdot)$ are all utility functions, which satisfy the following conditions: the first-order derivative is greater than zero and the second-order derivative is less than zero. The parameter $\beta$ is the subjective discount rate and $\alpha$ determines the relative importance of the intermediate consumption and the bequest. When $\alpha=0$, the expected utility only depends on the terminal wealth and the problem (7) is reduced to an asset allocation problem.

\section{The Optimal Portfolios}

In this section, we apply dynamic programming principle to obtain the HJB equation of the value function and study the optimal investment and consumption strategy under power utility and logarithm utility function. By using variable change technique, we obtain the closed-form solutions of the optimal portfolios.

The value function is defined as

$$
\begin{aligned}
& H(t, r, \eta, x) \\
& =\operatorname{Sup}_{\left(\pi_{t}, C_{t}\right) \in \Gamma} E\left[\alpha \int_{0}^{T} e^{-\beta t} U_{1}(C(t)) d t\right. \\
& +(1-\alpha) e^{-\beta T} U_{2}(X(T)) \mid \\
& \quad X(t)=x, r(t)=r, \eta(t)=\eta]
\end{aligned}
$$

with boundary condition $H(T, r, \eta, x)=(1-\alpha) e^{-\beta T} U_{2}(x)$.

Dynamic programming principle leads to the following HJB equation for $H(t, r, \eta, x)$ :

$$
\begin{aligned}
\operatorname{Sup}_{\left(\pi_{t}, C_{t}\right) \in \Gamma}\{ & H_{t}+(r x+\pi(t) k \eta-C(t)) H_{x} \\
& +\frac{1}{2} \pi^{2}(t) \sigma_{1}^{2} \eta H_{x x}+(\theta-c r) H_{r} \\
& +\frac{1}{2} \sigma_{0}^{2} r H_{r r}+(b-a \eta) H_{\eta}+\frac{1}{2} \sigma_{2}^{2} \eta H_{\eta \eta} \\
& \left.+\pi(t) \sigma_{1} \sigma_{2} H_{x \eta}+\alpha e^{-\beta t} U_{1}(C(t))\right\}=0,
\end{aligned}
$$

where $H_{t}, H_{x}, H_{x x}, H_{r}, H_{r r}, H_{\eta}, H_{\eta \eta}$, and $H_{x \eta}$ denote partial derivatives of first-order and second-order with respect to the time $t$, wealth process $X(t)$, interest rate $r(t)$, and volatility $\eta(t)$. We also use similar notation for higher derivatives and the derivatives of other functions.

The first-order maximizing conditions for the optimal investment and consumption strategy are

$$
\begin{gathered}
\pi^{*}(t)=-\frac{k}{\sigma_{1}^{2}} \cdot \frac{H_{x}}{H_{x x}}-\frac{\sigma_{2}}{\sigma_{1}} \cdot \frac{H_{x \eta}}{H_{x x}}, \\
U_{1}^{\prime}\left(C^{*}(t)\right)=\frac{H_{x}}{\alpha e^{-\beta t}} .
\end{gathered}
$$

Putting (10) in (9), we obtain the HJB equation as follows:

$$
\begin{aligned}
H_{t}+ & r x H_{x}+(\theta-c r) H_{r} \\
& +\frac{1}{2} \sigma_{0}^{2} r H_{r r}+(b-a \eta) H_{\eta} \\
& +\frac{1}{2} \sigma_{2}^{2} \eta H_{\eta \eta}-\frac{k^{2} \eta}{2 \sigma_{1}^{2}} \cdot \frac{H_{x}^{2}}{H_{x x}} \\
& -\frac{k \eta \sigma_{2}}{\sigma_{1}} \cdot \frac{H_{x} H_{x \eta}}{H_{x x}}-\frac{1}{2} \sigma_{2}^{2} \eta \frac{H_{x \eta}^{2}}{H_{x x}} \\
& -C^{*}(t) H_{x}+\alpha e^{-\beta t} U_{1}\left(C^{*}(t)\right)=0 .
\end{aligned}
$$


In the following subsection, we choose power utility and logarithm utility function as our analysis and apply variable change technique to solve (11).

3.1. Power Utility. Power utility is given by

$$
U_{1}(x)=U_{2}(x)=\frac{x^{\delta}}{\delta}, \quad \delta<1, \delta \neq 0,
$$

where $\delta$ is the risk aversion factor.

We conjecture a solution to (11) with the following form:

$$
H(t, r, \eta, x)=e^{-\beta t} \frac{x^{\delta}}{\delta} f(t, r, \eta), \quad f(T, r, \eta)=1-\alpha .
$$

Then the partial derivatives are as follows:

$$
\begin{gathered}
H_{t}=-\beta e^{-\beta t} \frac{x^{\delta}}{\delta} f+e^{-\beta t} \frac{x^{\delta}}{\delta} f_{t}, \\
H_{x x}=(\delta-1) e^{-\beta t} x^{\delta-2} f, \\
H_{x}=e^{-\beta t} x^{\delta-1} f, \\
H_{r}=e^{-\beta t} \frac{x^{\delta}}{\delta} f_{r}, \\
H_{r r}=e^{-\beta t} \frac{x^{\delta}}{\delta} f_{r r}, \\
H_{\eta}=e^{-\beta t} \frac{x^{\delta}}{\delta} f_{\eta}, \\
H_{\eta \eta}=e^{-\beta t} \frac{x^{\delta}}{\delta} f_{\eta \eta}, \\
H_{x \eta}=e^{-\beta t} x^{\delta-1} f_{\eta} .
\end{gathered}
$$

Therefore, the optimal consumption strategy is given by

$$
C^{*}(t)=\alpha^{-1 /(\delta-1)} f^{1 /(\delta-1)} x .
$$

Introducing (14) and (15) into (11), we obtain

$$
\begin{aligned}
e^{-\beta t} \frac{x^{\delta}}{\delta}\left[f_{t}\right. & +\left(r \delta-\beta-\frac{\delta k^{2} \eta}{2(\delta-1) \sigma_{1}^{2}}\right) f \\
& +(\theta-c r) f_{r}+\frac{1}{2} \sigma_{0}^{2} r f_{r r} \\
& +\left(b-a \eta-\frac{\delta k \eta \sigma_{2}}{(\delta-1) \sigma_{1}}\right) f_{\eta} \\
& +\frac{1}{2} \sigma_{2}^{2} \eta f_{\eta \eta}-\frac{\delta \sigma_{2}^{2} \eta}{2(\delta-1)} \frac{f_{\eta}^{2}}{f} \\
& \left.+(1-\delta) \alpha^{1 /(\delta-1)} f^{\delta /(\delta-1)}\right]=0
\end{aligned}
$$

Eliminating the dependence on $x$, we get

$$
\begin{aligned}
f_{t}+ & \left(r \delta-\beta-\frac{\delta k^{2} \eta}{2(\delta-1) \sigma_{1}^{2}}\right) f+(\theta-c r) f_{r} \\
& +\left(b-a \eta-\frac{\delta k \eta \sigma_{2}}{(\delta-1) \sigma_{1}}\right) f_{\eta}+\frac{1}{2} \sigma_{0}^{2} r f_{r r} \\
& +\frac{1}{2} \sigma_{2}^{2} \eta f_{\eta \eta}-\frac{\delta \sigma_{2}^{2} \eta}{2(\delta-1)} \frac{f_{\eta}^{2}}{f} \\
& +(1-\delta) \alpha^{1 /(\delta-1)} f^{\delta /(\delta-1)}=0 .
\end{aligned}
$$

Suppose that the solution structure of (17) is of the form

$$
f(t, r, \eta)=g(t, r, \eta)^{1-\delta}, \quad g(T, r, \eta)=(1-\alpha)^{1 /(\delta-1)} .
$$

Then, we have

$$
\begin{gathered}
f_{t}=(1-\delta) g^{-\delta} g_{t}, \\
f_{r}=(1-\delta) g^{-\delta} g_{r}, \\
f_{\eta}=(1-\delta) g^{-\delta} g_{\eta}, \\
f_{r r}=(1-\delta)(-\delta) g^{-\delta-1} g_{r}^{2}+(1-\delta) g^{-\delta} g_{r r}, \\
f_{\eta \eta}=(1-\delta)(-\delta) g^{-\delta-1} g_{\eta}^{2}+(1-\delta) g^{-\delta} g_{\eta \eta} .
\end{gathered}
$$

Substituting (19) into (17), after some simplification, we have

$$
\begin{aligned}
(1-\delta) g^{-\delta}[ & g_{t}+\left(\frac{\delta r-\beta}{1-\delta}+\frac{\delta}{(\delta-1)^{2}} \cdot \frac{k^{2} \eta}{2 \sigma_{1}^{2}}\right) g \\
& +(\theta-c r) g_{r}+\frac{1}{2} \sigma_{0}^{2} r g_{r r}-\frac{1}{2} \sigma_{0}^{2} r \delta \frac{g_{r}^{2}}{g} \\
& +\left(b-a \eta-\frac{\delta}{\delta-1} \cdot \frac{k \eta \sigma_{2}}{\sigma_{1}}\right) g_{\eta}+\frac{1}{2} \sigma_{2}^{2} \eta g_{\eta \eta} \\
& \left.+\alpha^{1 /(1-\delta)}\right]=0
\end{aligned}
$$

This leads to another equation for $g$ as

$$
\begin{aligned}
g_{t}+ & \left(\frac{\delta r-\beta}{1-\delta}+\frac{\delta}{(\delta-1)^{2}} \cdot \frac{k^{2} \eta}{2 \sigma_{1}^{2}}\right) g \\
& +(\theta-c r) g_{r}+\frac{1}{2} \sigma_{0}^{2} r g_{r r}-\frac{1}{2} \sigma_{0}^{2} r \delta \frac{g_{r}^{2}}{g} \\
& +\left(b-a \eta-\frac{\delta}{\delta-1} \cdot \frac{k \eta \sigma_{2}}{\sigma_{1}}\right) g_{\eta} \\
& +\frac{1}{2} \sigma_{2}^{2} \eta g_{\eta \eta}+\alpha^{1 /(1-\delta)}=0 .
\end{aligned}
$$


Equation (21) is a nonlinear second-order partial differential equation which is difficult to solve directly, for there exists the term $\alpha^{1 /(1-\delta)}$. Inspired by the paper of Liu [28], we assume that $g$ is of the following form:

$$
\begin{aligned}
g(t, r, \eta)= & \alpha^{1 /(1-\delta)} \int_{t}^{T} \hat{g}(u, r, \eta) d u \\
& +(1-\alpha)^{1 /(1-\delta)} \hat{g}(t, r, \eta) .
\end{aligned}
$$

One can prove that (21) is reduced to

$$
\begin{aligned}
\widehat{g}_{t}+ & \left(\frac{\delta r-\beta}{1-\delta}+\frac{\delta}{(\delta-1)^{2}} \cdot \frac{k^{2} \eta}{2 \sigma_{1}^{2}}\right) \widehat{g} \\
& +(\theta-c r) \widehat{g}_{r}+\frac{1}{2} \sigma_{0}^{2} r \widehat{g}_{r r}-\frac{1}{2} \sigma_{0}^{2} r \delta \frac{\widehat{g}_{r}^{2}}{\widehat{g}} \\
& +\left(b-a \eta-\frac{\delta}{\delta-1} \cdot \frac{k \eta \sigma_{2}}{\sigma_{1}}\right) \widehat{g}_{\eta} \\
& +\frac{1}{2} \sigma_{2}^{2} \eta \widehat{g}_{\eta \eta}=0, \quad \widehat{g}(T, r, \eta)=1 .
\end{aligned}
$$

In fact, if we define a differential operator $\nabla$ on any function $g$ by

$$
\begin{aligned}
\nabla g= & \left(\frac{\delta r-\beta}{1-\delta}+\frac{\delta}{(\delta-1)^{2}} \cdot \frac{k^{2} \eta}{2 \sigma_{1}^{2}}\right) g+(\theta-c r) g_{r} \\
& +\frac{1}{2} \sigma_{0}^{2} r g_{r r}-\frac{1}{2} \sigma_{0}^{2} r \delta \frac{g_{r}^{2}}{g} \\
& +\left(b-a \eta-\frac{\delta}{\delta-1} \cdot \frac{k \eta \sigma_{2}}{\sigma_{1}}\right) g_{\eta} \\
& +\frac{1}{2} \sigma_{2}^{2} \eta g_{\eta \eta} .
\end{aligned}
$$

Then (23) can be rewritten as

$$
\frac{\partial \widehat{g}}{\partial t}+\nabla \widehat{g}=0, \quad \widehat{g}(T, r, \eta)=1 .
$$

Furthermore, we find

$$
\begin{aligned}
\frac{\partial g}{\partial t}+\nabla g= & \frac{\partial}{\partial t}\left(\alpha^{1 /(1-\delta)} \int_{t}^{T} \hat{g}(u, r, \eta) d u\right) \\
& +\nabla\left(\alpha^{1 /(1-\delta)} \int_{t}^{T} \hat{g}(u, r, \eta) d u\right) \\
& +(1-\alpha)^{1 /(1-\delta)}\left(\frac{\partial}{\partial t} \widehat{g}(t, r, \eta)+\nabla \widehat{g}(t, r, \eta)\right) .
\end{aligned}
$$

Taking (25) into consideration, we have

$$
\begin{aligned}
\frac{\partial g}{\partial t}+\nabla g= & -\alpha^{1 /(1-\delta)} \hat{g}(t, r, \eta) \\
& +\alpha^{1 /(1-\delta)} \int_{t}^{T} \nabla \hat{g}(u, r, \eta) d u
\end{aligned}
$$

$$
\begin{aligned}
= & -\alpha^{1 /(1-\delta)} \widehat{g}(t, r, \eta) \\
& -\alpha^{1 /(1-\delta)} \int_{t}^{T} \frac{\partial \widehat{g}(u, r, \eta)}{\partial u} d u \\
= & -\alpha^{1 /(1-\delta)} \hat{g}(t, r, \eta) \\
& -\alpha^{1 /(1-\delta)}[\widehat{g}(T, r, \eta)-\widehat{g}(t, r, \eta)] \\
= & -\alpha^{1 /(1-\delta)} .
\end{aligned}
$$

Therefore, we obtain

$$
\frac{\partial g}{\partial t}+\nabla g+\alpha^{1 /(1-\delta)}=0, \quad g(T, r, \eta)=(1-\alpha)^{1 /(1-\delta)} .
$$

This is to say that (21) holds.

For (23), we can fit a solution of the following form:

$$
\widehat{g}(t, r, \eta)=\exp \{\phi(t) \eta+h(t) r+\Psi(t)\}
$$

with boundary condition $\phi(T)=h(T)=\Psi(T)=0$.

Introducing (29) into (23) yields

$$
\begin{aligned}
& \widehat{g}\left\{\eta \left[\phi^{\prime}(t)+\frac{\delta}{(\delta-1)^{2}} \cdot \frac{k^{2}}{2 \sigma_{1}^{2}}\right.\right. \\
& \left.\quad-\left(a+\frac{\delta}{\delta-1} \cdot \frac{k \sigma_{2}}{\sigma_{1}}\right) \phi(t)+\frac{1}{2} \sigma_{2}^{2} \phi^{2}(t)\right] \\
& +r\left[h^{\prime}(t)+\frac{\delta}{1-\delta}-c h(t)+\frac{1}{2}(1-\delta) \sigma_{0}^{2} h^{2}(t)\right] \\
& \left.+\Psi^{\prime}(t)+b \phi(t)+\theta h(t)-\frac{\beta}{1-\delta}\right\}=0 .
\end{aligned}
$$

We can decompose (30) into the following three equations in order to eliminate the dependence on $r$ and $\eta$ :

$$
\begin{gathered}
\phi^{\prime}(t)+\frac{\delta}{(\delta-1)^{2}} \cdot \frac{k^{2}}{2 \sigma_{1}^{2}} \\
-\left(a+\frac{\delta}{\delta-1} \cdot \frac{k \sigma_{2}}{\sigma_{1}}\right) \phi(t) \\
+\frac{1}{2} \sigma_{2}^{2} \phi^{2}(t)=0, \quad \phi(T)=0, \\
h^{\prime}(t)+\frac{\delta}{1-\delta}-c h(t)+\frac{1}{2}(1-\delta) \sigma_{0}^{2} h^{2}(t)=0, \quad h(T)=0, \\
\Psi^{\prime}(t)+b \phi(t)+\theta h(t)-\frac{\beta}{1-\delta}=0, \quad \Psi(T)=0 .
\end{gathered}
$$

Rewritting (31), we have

$$
\begin{gathered}
\phi^{\prime}(t)=-\frac{1}{2} \sigma_{2}^{2}\left[\phi^{2}(t)-\frac{2}{\sigma_{2}^{2}}\left(a+\frac{\delta}{\delta-1} \cdot \frac{k \sigma_{2}}{\sigma_{1}}\right) \phi(t)\right. \\
\left.+\frac{2}{\sigma_{2}^{2}} \cdot \frac{\delta}{(\delta-1)^{2}} \cdot \frac{k^{2}}{2 \sigma_{1}^{2}}\right] .
\end{gathered}
$$


Let $\Delta_{\phi}$ denote the discriminant of the quadratic equation

$$
\begin{gathered}
\phi^{2}(t)-\frac{2}{\sigma_{2}^{2}}\left(a+\frac{\delta}{\delta-1} \cdot \frac{k \sigma_{2}}{\sigma_{1}}\right) \phi(t) \\
+\frac{2}{\sigma_{2}^{2}} \cdot \frac{\delta}{(\delta-1)^{2}} \cdot \frac{k^{2}}{2 \sigma_{1}^{2}}=0
\end{gathered}
$$

and then we have

$$
\Delta_{\phi}=\frac{4}{\sigma_{2}^{4}}\left[\frac{-a^{2}}{\delta-1}+\frac{\delta}{\delta-1}\left(a+\frac{k \sigma_{2}}{\sigma_{1}}\right)^{2}\right] .
$$

Suppose that $\Delta_{\phi}>0$, that is,

$$
\delta<\frac{a^{2}}{\left(a+\left(k \sigma_{2} / \sigma_{1}\right)\right)^{2}}<1 .
$$

Under the condition (37), integrating (34) on both sides with respect to $t$, we obtain

$$
\frac{1}{\lambda_{1}-\lambda_{2}} \int_{t}^{T}\left(\frac{1}{\phi(t)-\lambda_{1}}-\frac{1}{\phi(t)-\lambda_{2}}\right) d \phi(t)=-\frac{1}{2} \sigma_{2}^{2}(T-t),
$$

where $\lambda_{1}$ and $\lambda_{2}$ are two real roots of (35), namely,

$$
\begin{aligned}
\lambda_{1,2}= & \frac{1}{\sigma_{2}^{2}}\left(a+\frac{\delta}{\delta-1} \cdot \frac{k \sigma_{2}}{\sigma_{1}}\right) \\
& \pm \frac{1}{\sigma_{2}^{2}} \sqrt{\frac{-a^{2}}{\delta-1}+\frac{\delta}{\delta-1}\left(a+\frac{k \sigma_{2}}{\sigma_{1}}\right)^{2}} .
\end{aligned}
$$

Solving (38) with boundary condition $\phi(T)=0$, we derive

$$
\phi(t)=\frac{\lambda_{1} \lambda_{2}\left(1-\exp \left\{-(1 / 2) \sigma_{2}^{2}\left(\lambda_{1}-\lambda_{2}\right)(T-t)\right\}\right)}{\lambda_{1}-\lambda_{2} \exp \left\{-(1 / 2) \sigma_{2}^{2}\left(\lambda_{1}-\lambda_{2}\right)(T-t)\right\}} .
$$

For (32), using the same analysis as $\phi(t)$, we obtain

$$
h(t)=\frac{\lambda_{3} \lambda_{4}\left(1-\exp \left\{-((1-\delta) / 2) \sigma_{0}^{2}\left(\lambda_{3}-\lambda_{4}\right)(T-t)\right\}\right)}{\lambda_{3}-\lambda_{4} \exp \left\{-((1-\delta) / 2) \sigma_{0}^{2}\left(\lambda_{3}-\lambda_{4}\right)(T-t)\right\}}
$$

under the condition

$$
\delta<\frac{c^{2}}{2 \sigma_{0}^{2}}
$$

where $\lambda_{3}$ and $\lambda_{4}$ are two real roots of the equation

$$
h^{2}(t)-\frac{2 c}{(1-\delta) \sigma_{0}^{2}} h(t)+\frac{\delta}{1-\delta} \cdot \frac{2}{(1-\delta) \sigma_{0}^{2}}=0 .
$$

Easy calculation leads to

$$
\lambda_{3,4}=\frac{1}{(1-\delta) \sigma_{0}^{2}} \pm \frac{\sqrt{c^{2}-2 \delta \sigma_{0}^{2}}}{(1-\delta) \sigma_{0}^{2}}
$$

For (33), we have

$$
\Psi(t)=b \int_{t}^{T} \phi(s) d s+\theta \int_{t}^{T} h(s) d s-\frac{\beta}{1-\delta}(T-t) .
$$

Combining (37) with (42), we find that the risk aversion factor $\delta$ should satisfy

$$
\delta<\operatorname{Min}\left\{\frac{a^{2} \sigma_{1}^{2}}{\left(a \sigma_{1}+k \sigma_{2}\right)^{2}}, \frac{c^{2}}{2 \sigma_{0}^{2}}\right\}, \quad \delta \neq 0
$$

According to (14) and (19), we have

$$
\frac{H_{x}}{H_{x x}}=\frac{1}{\delta-1} x, \quad \frac{H_{x \eta}}{H_{x x}}=-\frac{g_{\eta}}{g} x .
$$

Finally, taking (10) and (15) into consideration, we obtain the optimal investment and consumption strategy under the power utility function.

Proposition 2. If utility function $U_{1}(x)=U_{2}(x)=x^{\delta} / \delta$, with the conditions $\delta<\operatorname{Min}\left\{a^{2} \sigma_{1}^{2} /\left(a \sigma_{1}+k \sigma_{2}\right)^{2}, c^{2} / 2 \sigma_{0}^{2}\right\}$ and $\delta \neq 0$, the optimal investment and consumption strategy of the problem (7) is given by

$$
\begin{gathered}
\pi_{t}^{*}=\frac{k}{(1-\delta) \sigma_{1}^{2}} X_{t}+\frac{\sigma_{2}}{\sigma_{1}} \cdot \frac{g_{\eta}}{g} X_{t}, \\
C^{*}(t)=\alpha^{1 /(1-\delta)} g^{-1} X_{t},
\end{gathered}
$$

where

$$
\begin{aligned}
g= & g(t, r, \eta) \\
= & \alpha^{1 /(1-\delta)} \int_{t}^{T} \exp \{\phi(s) \eta+h(s) r+\Psi(s)\} d s \\
& +(1-\alpha)^{1 /(1-\delta)} \exp \{\phi(t) \eta+h(t) r+\Psi(t)\} .
\end{aligned}
$$

In addition, $\phi(t), h(t)$, and $\Psi(t)$ are determined by (40), (41) and (45), respectively.

In order to compare the results obtained by Proposition 2 with those in the existing literatures, we consider the following five special cases in the power utility case.

Special Case 1. If $\theta=c=\sigma_{0}=0$, this is to say that risk-free interest rate $r(t)$ will become a constant. Then the problem (7) is degenerated into an investment and consumption problem with stochastic volatility. After easy calculation, we obtain

$$
\begin{gathered}
\pi_{t}^{*}=\frac{k}{(1-\delta) \sigma_{1}^{2}} X_{t}+\frac{\sigma_{2}}{\sigma_{1}} \cdot \frac{g_{\eta}}{g} X_{t}, \\
C^{*}(t)=\alpha^{1 /(1-\delta)} g^{-1} X_{t}
\end{gathered}
$$


under the conditions $\delta<a^{2} \sigma_{1}^{2} /\left(a \sigma_{1}+k \sigma_{2}\right)^{2}$ and $\delta \neq 0$, where

$$
\begin{gathered}
\lambda_{1,2}=\frac{1}{\sigma_{2}^{2}}\left(a+\frac{\delta}{\delta-1} \cdot \frac{k \sigma_{2}}{\sigma_{1}}\right) \\
\pm \frac{1}{\sigma_{2}^{2}} \sqrt{\frac{-a^{2}}{\delta-1}+\frac{\delta}{\delta-1}\left(a+\frac{k \sigma_{2}}{\sigma_{1}}\right)^{2}} \\
\phi(t)=\frac{\lambda_{1} \lambda_{2}\left(1-\exp \left\{-(1 / 2) \sigma_{2}^{2}\left(\lambda_{1}-\lambda_{2}\right)(T-t)\right\}\right)}{\lambda_{1}-\lambda_{2} \exp \left\{-(1 / 2) \sigma_{2}^{2}\left(\lambda_{1}-\lambda_{2}\right)(T-t)\right\}} \\
h(t)=\frac{\delta}{1-\delta}(T-t), \\
g=g(t, r, \eta)=b \int_{t}^{T} \phi(s) d s-\frac{\beta}{1-\delta}(T-t), \\
=\alpha^{1 /(1-\delta)} \int_{t}^{T} \exp \{\phi(s) \eta+h(s) r+\Psi(s)\} d s \\
+(1-\alpha)^{1 /(1-\delta)} \exp \{\phi(t) \eta+h(t) r+\Psi(t)\}
\end{gathered}
$$

Special Case 2. If $a=b=\sigma_{2}=0$, namely, volatility term $\eta(t)$ reduces to a constant, then the problem (7) becomes an investment and consumption problem with stochastic interest rate. Therefore, we have

$$
\pi_{t}^{*}=\frac{k}{(1-\delta) \sigma_{1}^{2}} X_{t}, \quad C^{*}(t)=\alpha^{1 /(1-\delta)} g^{-1} X_{t}
$$

under the conditions $\delta<\operatorname{Min}\left\{c^{2} / 2 \sigma_{0}^{2}, 1\right\}$ and $\delta \neq 0$, where

$$
\begin{aligned}
g= & g(t, r, \eta) \\
= & \alpha^{1 /(1-\delta)} \int_{t}^{T} \exp \{\phi(s) \eta+h(s) r+\Psi(s)\} d s \\
& +(1-\alpha)^{1 /(1-\delta)} \exp \{\phi(t) \eta+h(t) r+\Psi(t)\}
\end{aligned}
$$

with

$$
\begin{gathered}
\phi(t)=\frac{\delta}{(\delta-1)^{2}} \cdot \frac{k^{2}}{2 \sigma_{1}^{2}}(T-t), \\
\Psi(t)=\theta \int_{t}^{T} h(s) d s-\frac{\beta}{1-\delta}(T-t), \\
\lambda_{3,4}=\frac{1}{(1-\delta) \sigma_{0}^{2}} \pm \frac{\sqrt{c^{2}-2 \delta \sigma_{0}^{2}}}{(1-\delta) \sigma_{0}^{2}}, \\
h(t)=\frac{\lambda_{3} \lambda_{4}\left(1-\exp \left\{-((1-\delta) / 2) \sigma_{0}^{2}\left(\lambda_{3}-\lambda_{4}\right)(T-t)\right\}\right)}{\lambda_{3}-\lambda_{4} \exp \left\{-((1-\delta) / 2) \sigma_{0}^{2}\left(\lambda_{3}-\lambda_{4}\right)(T-t)\right\}} .
\end{gathered}
$$

Special Case 3. If $\theta=c=\sigma_{0}=0$ and $a=b=\sigma_{2}=0$, that is, risk-free interest rate $r(t)$ and volatility term $\eta(t)$ of the stock become the constants, then the problem (7) is reduced to an investment and consumption problem with constant interest rate and constant volatility rate. In addition, we get

$$
\pi_{t}^{*}=\frac{k}{(1-\delta) \sigma_{1}^{2}} X_{t}, \quad C^{*}(t)=\alpha^{1 /(1-\delta)} g^{-1} X_{t}
$$

under the conditions $\delta<1$ and $\delta \neq 0$, where

$$
\begin{gathered}
g=g(t, r, \eta) \\
=\alpha^{1 /(1-\delta)} \frac{e^{\omega(T-t)}-1}{\omega}+(1-\alpha)^{1 /(1-\delta)} e^{\omega(T-t)}, \\
\omega=\frac{\delta \eta}{(\delta-1)^{2}} \cdot \frac{k^{2}}{2 \sigma_{1}^{2}}+\frac{\delta r}{1-\delta}-\frac{\beta}{1-\delta} .
\end{gathered}
$$

Special Case 4. If $\alpha=0$ and $\beta=0$, the problem (7) is degenerated into a dynamic asset allocation problem with stochastic interest rate and stochastic volatility. Therefore, the optimal investment strategy is given by

$$
\pi_{t}^{*}=\frac{k}{(1-\delta) \sigma_{1}^{2}} X_{t}+\frac{\sigma_{2}}{\sigma_{1}} \cdot \phi(t) X_{t}
$$

with the conditions $\delta<\operatorname{Min}\left\{a^{2} \sigma_{1}^{2} /\left(a \sigma_{1}+k \sigma_{2}\right)^{2}, c^{2} / 2 \sigma_{0}^{2}\right\}$ and $\delta \neq 0$, where

$$
\begin{gathered}
\lambda_{1,2}=\frac{1}{\sigma_{2}^{2}}\left(a+\frac{\delta}{\delta-1} \cdot \frac{k \sigma_{2}}{\sigma_{1}}\right) \\
\pm \frac{1}{\sigma_{2}^{2}} \sqrt{\frac{-a^{2}}{\delta-1}+\frac{\delta}{\delta-1}\left(a+\frac{k \sigma_{2}}{\sigma_{1}}\right)^{2}}, \\
\phi(t)=\frac{\lambda_{1} \lambda_{2}\left(1-\exp \left\{-(1 / 2) \sigma_{2}^{2}\left(\lambda_{1}-\lambda_{2}\right)(T-t)\right\}\right)}{\lambda_{1}-\lambda_{2} \exp \left\{-(1 / 2) \sigma_{2}^{2}\left(\lambda_{1}-\lambda_{2}\right)(T-t)\right\}} .
\end{gathered}
$$

Special Case 5. If $\delta=0$, power utility will degenerate into a logarithmic utility. After easy calculation, we get

$$
\begin{gathered}
\phi(t)=0, \quad h(t)=0, \quad \Psi(t)=-\beta(T-t), \\
g=g(t, r, \eta)=\frac{\alpha}{\beta}\left(1-e^{-\beta(T-t)}\right)+(1-\alpha) e^{-\beta(T-t)} .
\end{gathered}
$$

As a result, the optimal investment and consumption strategy of the problem (7) in the logarithm utility case is given by

$$
\begin{gathered}
\pi_{t}^{*}=\frac{k}{\sigma_{1}^{2}} X_{t}, \\
C^{*}(t)=\frac{\alpha}{(\alpha / \beta)\left(1-e^{-\beta(T-t)}\right)+(1-\alpha) e^{-\beta(T-t)}} X_{t} .
\end{gathered}
$$

The above results are in accordance with those under the logarithm utility maximizing criterion in the following subsection. 
3.2. Logarithm Utility. Assume that the logarithm utility is given by

$$
U_{1}(x)=U_{2}(x)=\ln x .
$$

Then we conjecture a solution to (11) with the following form;

$$
H(t, r, \eta, x)=u(t) e^{-\beta t} \ln x+v(t, r, \eta),
$$

where boundary conditions are given by $u(T)=1-\alpha$ and $v(T, r, \eta)=0$.

Putting (62) in (11), we get

$$
\begin{aligned}
& e^{-\beta x} \ln x\left(u^{\prime}(t)-\beta u(t)+\alpha\right)+v_{t} \\
& +r u(t) e^{-\beta t}+(\theta-c r) v_{r}+\frac{1}{2} \sigma_{0}^{2} r v_{r r} \\
& +(b-a \eta) v_{\eta}+\frac{1}{2} \sigma_{2}^{2} \eta v_{\eta \eta} \\
& +\frac{k^{2} \eta}{2 \sigma_{1}^{2}} u(t) e^{-\beta t}+(\ln \alpha-1) \alpha e^{-\beta t} \\
& -\alpha e^{-\beta t} \ln u(t)=0 .
\end{aligned}
$$

Eliminating the dependence on $x$, we have

$$
\begin{aligned}
& u^{\prime}(t)-\beta u(t)+\alpha=0, \quad u(T)=1-\alpha, \\
& v_{t}+r u(t) e^{-\beta t}+(\theta-c r) v_{r} \\
& +\frac{1}{2} \sigma_{0}^{2} r v_{r r}+(b-a \eta) v_{\eta}+\frac{1}{2} \sigma_{2}^{2} \eta v_{\eta \eta} \\
& +\frac{k^{2} \eta}{2 \sigma_{1}^{2}} u(t) e^{-\beta t}+(\ln \alpha-1) \alpha e^{-\beta t} \\
& -\alpha e^{-\beta t} \ln u(t)=0 .
\end{aligned}
$$

Solving (64), we get

$$
u(t)=(1-\alpha) e^{-\beta(T-t)}-\frac{\alpha}{\beta}\left(e^{-\beta(T-t)}-1\right) .
$$

Suppose that the solution to (65) is of the structure

$$
\begin{gathered}
v(t, r, \eta)=D(t)+E(t) r+F(t) \eta, \\
D(T)=E(T)=F(T)=0 .
\end{gathered}
$$

Putting (67) in (65), we derive

$$
\begin{aligned}
& r\left[E^{\prime}(t)-c E(t)+u(t) e^{-\beta t}\right] \\
& +\eta\left[F^{\prime}(t)-a F(t)+\frac{k^{2}}{2 \sigma_{1}^{2}} u(t) e^{-\beta t}\right] \\
& +D^{\prime}(t)+\theta E(t)+b F(t)+(\ln \alpha-1) \alpha e^{-\beta t} \\
& -\alpha e^{-\beta t} \ln u(t)=0 .
\end{aligned}
$$

By matching coefficients, we obtain the following three equations:

$$
\begin{gathered}
E^{\prime}(t)-c E(t)+u(t) e^{-\beta t}=0, \quad E(T)=0, \\
F^{\prime}(t)-a F(t)+\frac{k^{2}}{2 \sigma_{1}^{2}} u(t) e^{-\beta t}=0, \quad F(T)=0, \\
D^{\prime}(t)+\theta E(t)+b F(t)+(\ln \alpha-1) \alpha e^{-\beta t} \\
-\alpha e^{-\beta t} \ln u(t)=0, \quad D(T)=0 .
\end{gathered}
$$

Solving the above three equations (69)-(71), we obtain

$$
\begin{aligned}
E(t)= & \frac{\alpha+\alpha \beta-\beta}{\beta c} e^{-\beta T}\left(e^{-c(T-t)}-1\right) \\
& -\frac{\alpha}{\beta(\beta+c)} e^{-\beta T}\left(e^{-c(T-t)}-e^{\beta(T-t)}\right) \\
F(t)= & \frac{k^{2}}{2 \sigma_{1}^{2}} \cdot \frac{\alpha \beta+\alpha-\beta}{a \beta} e^{-\beta T}\left(e^{-a(T-t)}-1\right) \\
& -\frac{k^{2}}{2 \sigma_{1}^{2}} \cdot \frac{\alpha}{(a+\beta) \beta} e^{-\beta T}\left(e^{-a(T-t)}-e^{\beta(T-t)}\right) \\
D(t)= & \theta \int_{t}^{T} E(s) d s+b \int_{t}^{T} F(s) d s \\
& +(\ln \alpha-1) \alpha \int_{t}^{T} e^{-\beta s} d s-\alpha \int_{t}^{T} e^{-\beta s} \ln u(s) d s .
\end{aligned}
$$

Taking (62) into considering, we obtain

$$
\frac{H_{x}}{H_{x x}}=-x, \quad \frac{H_{x \eta}}{H_{x x}}=0, \quad C^{*}(t)=\frac{\alpha}{u(t)} x .
$$

Therefore, we can summarize the optimal investment and consumption strategy in the logarithm utility case as the following proposition.

Proposition 3. If utility function is given by $U_{1}(x)=U_{2}(x)=$ $\ln x$, the optimal investment and consumption strategy of the problem (7) is

$$
\begin{gathered}
\pi^{*}(t)=\frac{k}{\sigma_{1}^{2}} X_{t}, \\
C^{*}(t)=\frac{\alpha}{(1-\alpha) e^{-\beta(T-t)}-(\alpha / \beta)\left(e^{-\beta(T-t)}-1\right)} X_{t} .
\end{gathered}
$$

Remark 4. According to Proposition 3, we find the following. (i) The parameters $\theta, c$, and $\sigma_{1}$ in the stochastic interest rate model have no influence on $\pi^{*}(t)$ and $C^{*}(t)$, (ii) The parameters $b, a$, and $\sigma_{2}$ in the stochastic volatility model have no impact on $\pi^{*}(t)$ and $C^{*}(t)$ as well, (iii) The optimal amount $\pi^{*}(t)$ invested in the stock depends only on the parameters $k$ and $\sigma_{1}$. In addition, $\pi^{*}(t)$ increases with respect to $k$ and decreases with respect to $\sigma_{1}$, (iv) The optimal 


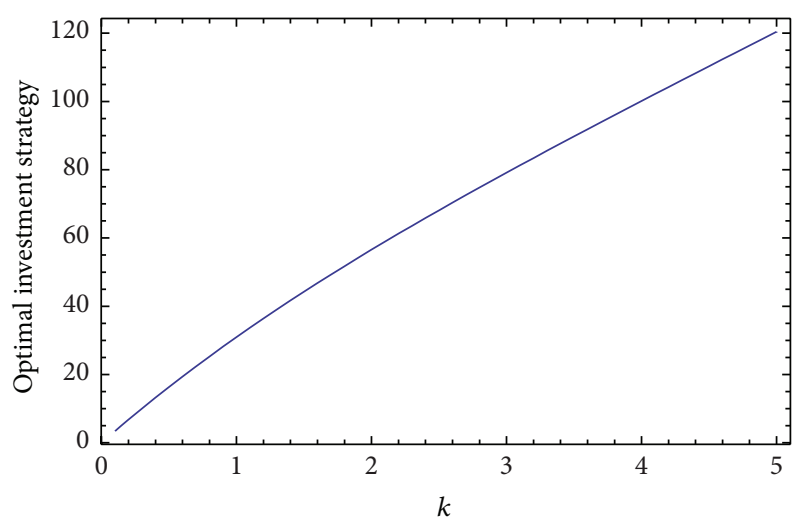

(a)

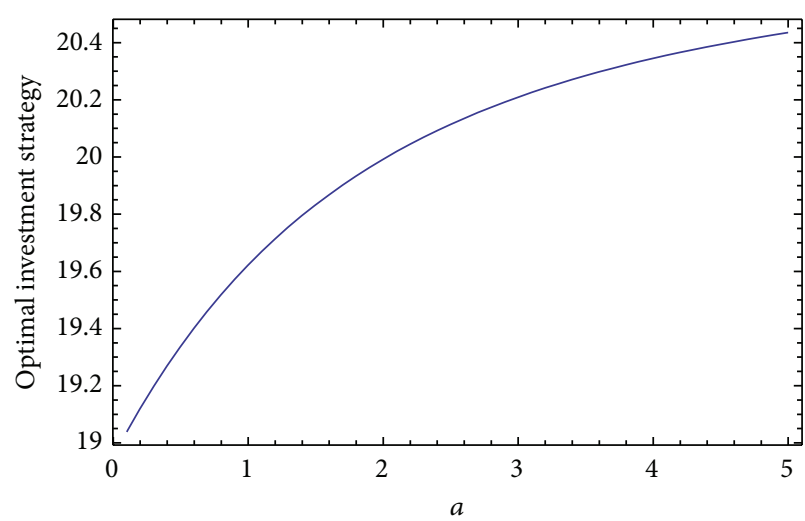

(b)

FIgURE 1: The impact of $k$ and $a$ on the optimal investment strategy.

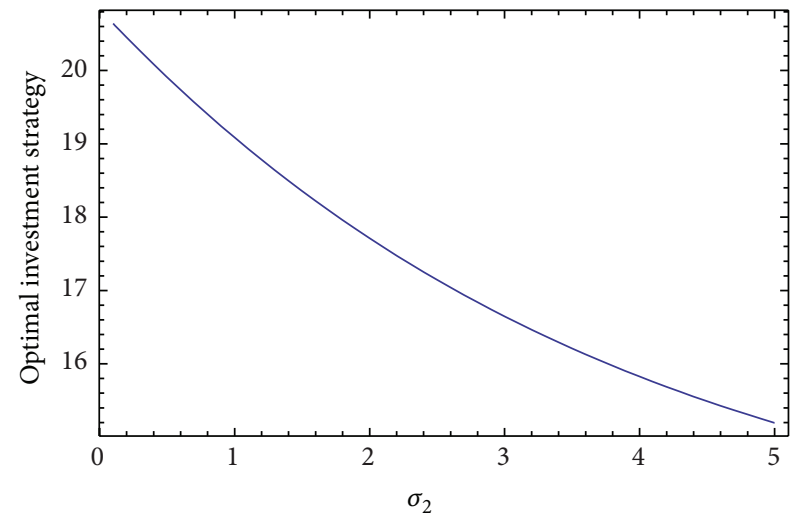

(a)

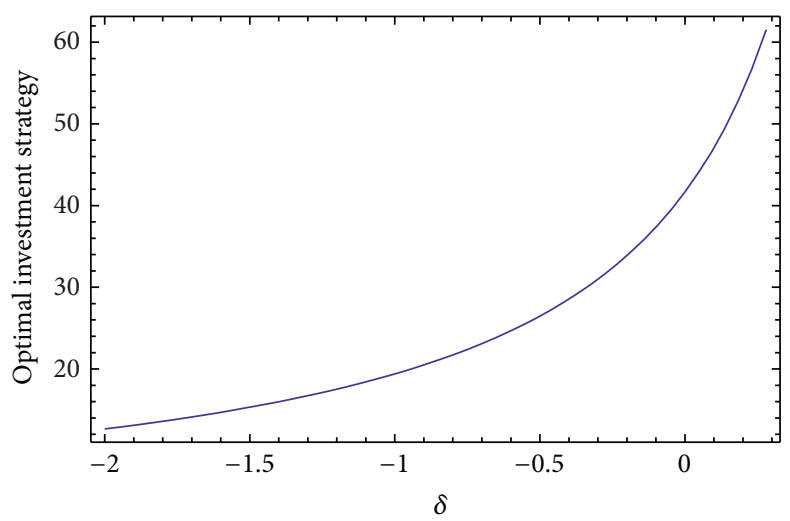

(b)

FIGURE 2: The impact of $\sigma_{2}$ and $\delta$ on the optimal investment strategy.

consumption rate $C^{*}(t)$ increases with respect to $\alpha$, for we can obtain

$$
\frac{d C^{*}(t)}{d \alpha}=\frac{e^{-\beta(T-t)}}{\left[(1-\alpha) e^{-\beta(T-t)}-(\alpha / \beta)\left(e^{-\beta(T-t)}-1\right)\right]^{2}} X_{t}>0 .
$$

\section{Numerical Analysis}

In this section, we will provide a numerical example to illustrate how market parameters affect the dynamic behavior of the optimal investment and consumption strategy in the power utility case. Throughout the numerical analysis, unless otherwise stated, the basic parameters are given by $c=0.15$, $\sigma_{0}=0.2, \theta=0.25, r(0)=0.05, \sigma_{1}=1.2, k=0.6, \eta(0)=0.36$, $\sigma_{2}=0.8, b=1.0, a=0.6, t=0, T=1, \alpha=0.4, \beta=0.1$, $\delta=-1$, and $x_{0}=100$.

4.1. The Impact on the Optimal Investment Strategy. It can be seen from Figures 1 and 2 that we can summarize the effect of market parameters on the optimal investment strategy and provide some economic implications. (a1) The optimal investment strategy $\pi^{*}(t)$ increases with respect to the parameter $k$. Recalling (3), $k \eta(t)$ can be taken as the appreciation rate of the stock. Hence, as the parameter $k$ increases, then the expected income of the stock increases, which leads to the fact that the investor wishes to invest more amount of his wealth in the stock.

(a2) $\pi^{*}(t)$ increases with respect to the parameter $a$. The larger the value of $a$, the smaller the expected value of $\eta(t)$. This leads to the fact that the expected appreciation rate of the stock becomes less and the volatility of the stock will become much smaller. Due to the much less risk of the investment, the investor would invest more money in the stock.

(a3) $\pi^{*}(t)$ is decreasing in $\sigma_{2}$. In fact, the larger the value of $\sigma_{2}$, the higher the volatility term of $\eta(t)$, which leads to that the volatility of the stock is increasing. Therefore, the risk of investment is increasing accordingly and the investor invests the less amount in the stock.

(a4) $\pi^{*}(t)$ is increasing in $\delta$. According to the condition of Proposition 2, the value of $\delta$ should be less than 0.28 . 


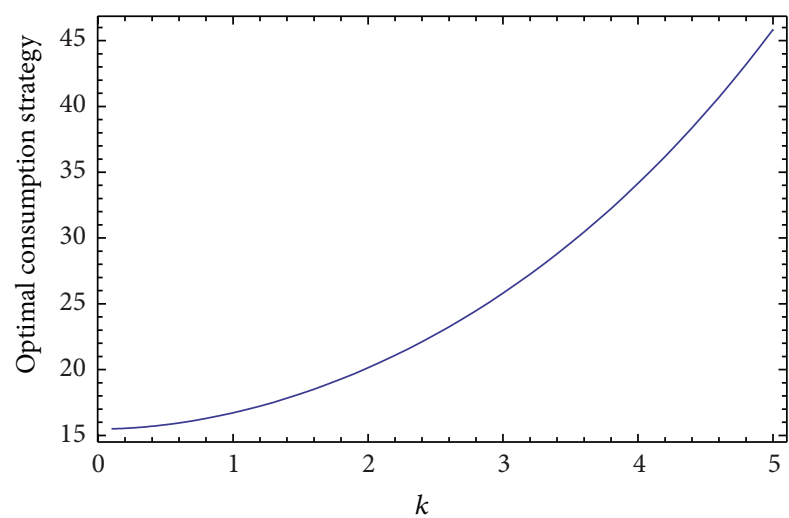

(a)

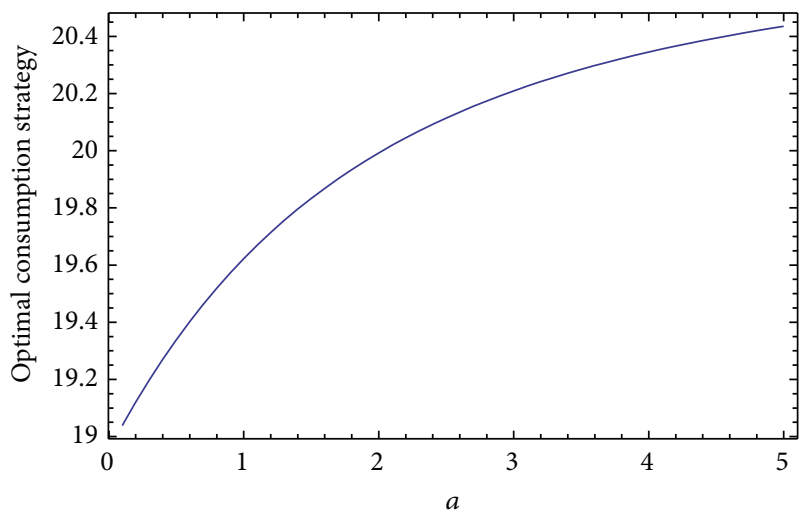

(b)

Figure 3: The impact of $k$ and $a$ on the optimal consumption strategy.

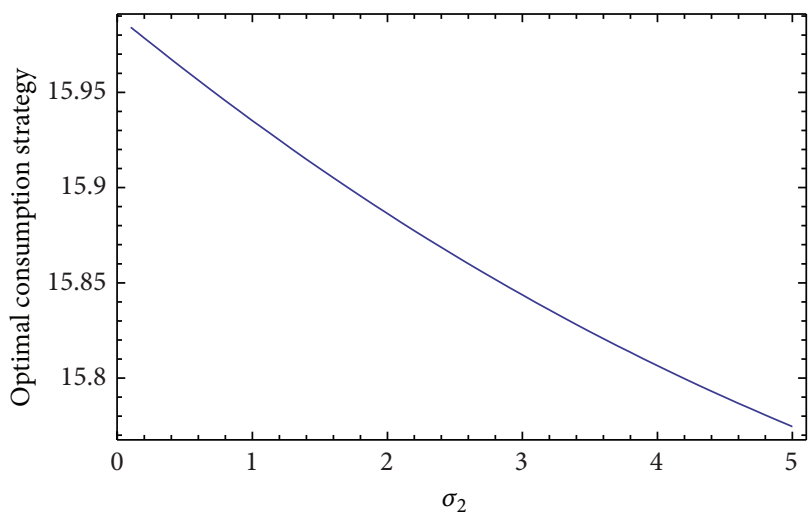

(a)

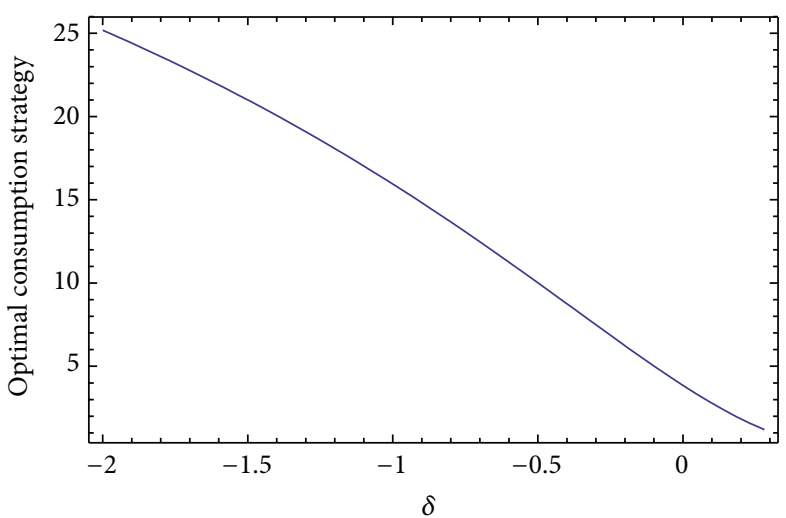

(b)

FIgURE 4: The impact of $\sigma_{2}$ and $\delta$ on the optimal consumption strategy.

In the power utility case, the coefficient of risk aversion is denoted by $1-\delta$. It indicates that the investor can take less risk as the parameter $\delta$ is increasing. Therefore, the larger the value of $\delta$, the more amount the investor wishes to invest in the stock. On the other hand, when $\delta=0$, power utility is degenerated to logarithm utility. Therefore, we can summarize that the optimal investment strategy in the power utility case will be less than that in the logarithm utility case when $\delta<0$, while the optimal investment policy for power utility will be more than that for logarithm utility when $0<\delta<0.28$.

4.2. The Impact on the Optimal Consumption Strategy. From Figures 3 and 4, we can summarize the following conclusions.

(b1) The optimal consumption strategy $C^{*}(t)$ increases with respect to the parameter $k$. In fact, when the value of $k$ becomes larger, the expected appreciation of the stock becomes larger as well. Therefore, the wealth of the investor will become much larger, which leads to the fact that the investor can consume the more amount of the wealth.

(b2) $C^{*}(t)$ is slowly decreasing in $a$. As the value of $a$ is increasing, the expected value of $\eta(t)$ is decreasing. It leads to the fact that the expected value of the wealth is decreasing. Therefore, the investor should consume less money.

(b3) $C^{*}(t)$ will slowly decrease as the parameter $\sigma_{2}$ increases. As a matter of fact, the bigger the value of $\sigma_{2}$, the less the amount invested in the stock. It means that the wealth of the investor is decreasing. Therefore, the investor should keep less consumption.

(b4) $C^{*}(t)$ will decrease with the parameter $\delta$. That is to say, the bigger the value of $\delta$, the less the risk aversion the investor needs to take and the less the investor can consume. Moreover, we conclude that the amount the investor can consume in the power utility case is more than that in the logarithm utility case when $\delta<0$, while the investor with power utility preference can consume less amount than the one with logarithm utility preference when $0<\delta<0.28$. 


\section{Conclusions}

In this paper, we investigate an investment and consumption problem with stochastic interest rate and stochastic volatility, in which interest rate dynamic is assumed to be driven by the CIR model and the volatility of stock price is described by Heston's stochastic volatility model. The financial market consists of one risk-free asset and one risky asset. Our objective is to maximize the expected discounted utility of consumption and terminal wealth in the fixed finite investment horizon. By applying dynamic programming principle, we obtain the explicit expressions of the optimal investment and consumption strategies in the power and logarithm utility cases. We have also given a numerical example to illustrate the results obtained and analyzed the effect of market parameters on the optimal trading strategies.

In recent years, there has been much attention to the portfolio selection problems in stochastic environments. This is due to the facts that risk-free interest rate and volatility of the stock is not always fixed in most of real-world situations and the optimal portfolios in stochastic environments will make it more practical. Under some special assumptions, we obtain the closed-form solutions of the optimal trading strategies in stochastic interest rate and stochastic volatility situations. These assumptions include the following: (i) interest rate has no correlation with risky asset dynamics; (ii) Heston's stochastic volatility and risky asset dynamics are driven by the same Brownian motion; (iii) stock price is assumed to follow geometric Brownian motion, which is a special case of the constant elasticity of variance (CEV) model. If we relax these assumptions and consider some more general cases, it will lead to more sophisticated HJB equations and will have no ways to get the closed-form solutions of the optimal trading strategy. In future research, we will explore new approaches to tackle these problems.

\section{Acknowledgments}

The author is very grateful to many anonymous reviewers for their insightful comments and suggestions. This research is supported by the Higher School Science and Technology Development Foundation of Tianjin (no. 20100821) and the Humanities and Social Science Research Youth Foundation of Ministry of Education (no. 11YJC790006).

\section{References}

[1] R. C. Merton, "Lifetime portfolio selection under uncertainty: the continuous-time case," The Review of Economics and Statistics, vol. 51, no. 3, pp. 247-257, 1969.

[2] R. C. Merton, "Optimum consumption and portfolio rules in a continuous-time model," Journal of Economic Theory, vol. 3, no. 4, pp. 373-413, 1971.

[3] W. H. Fleming and T. Zariphopoulou, "An optimal investment/ consumption model with borrowing," Mathematics of Operations Research, vol. 16, no. 4, pp. 802-822, 1991.

[4] J.-L. Vila and T. Zariphopoulou, "Optimal consumption and portfolio choice with borrowing constraints," Journal of Economic Theory, vol. 77, no. 2, pp. 402-431, 1997.
[5] D. Duffie, W. Fleming, H. M. Soner, and T. Zariphopoulou, "Hedging in incomplete markets with HARA utility," Journal of Economic Dynamics \& Control, vol. 21, no. 4-5, pp. 753-782, 1997.

[6] B. Dumas and E. Luciano, "An exact solution to a dynamic portfolio choice problem under transactions costs," The Journal of Finance, vol. 46, no. 2, pp. 577-595, 1991.

[7] S. E. Shreve and H. M. Soner, "Optimal investment and consumption with transaction costs," The Annals of Applied Probability, vol. 4, no. 3, pp. 609-692, 1994.

[8] H. Liu and M. Loewenstein, "Optimal portfolio selection with transaction costs and finite horizons," Review of Financial Studies, vol. 15, no. 3, pp. 805-835, 2002.

[9] M. Dai, L. Jiang, P. Li, and F. Yi, "Finite horizon optimal investment and consumption with transaction costs," SIAM Journal on Control and Optimization, vol. 48, no. 2, pp. 1134-1154, 2009.

[10] T. S. Y. Ho and S. B. Lee, "Term structure movements and pricing interest contingent claims," Journal of Finance, vol. 41, no. 5, pp. 1011-1029, 1986.

[11] O. Vasicek, "An equilibrium characterization of the term structure," Journal of Financial Economics, vol. 5, no. 2, pp. 177-188, 1977.

[12] J. C. Cox, J. E. Ingersoll, Jr., and S. A. Ross, "A theory of the term structure of interest rates," Econometrica. Journal of the Econometric Society, vol. 53, no. 2, pp. 385-407, 1985.

[13] R. Korn and H. Kraft, "A stochastic control approach to portfolio problems with stochastic interest rates," SIAM Journal on Control and Optimization, vol. 40, no. 4, pp. 1250-1269, 2001/02.

[14] M. Grasselli, "A stability result for the HARA class with stochastic interest rates," Insurance: Mathematics \& Economics, vol. 33, no. 3, pp. 611-627, 2003.

[15] G. Deelstra, M. Grasselli, and P.-F. Koehl, "Optimal investment strategies in the presence of a minimum guarantee," Insurance: Mathematics \& Economics, vol. 33, no. 1, pp. 189-207, 2003.

[16] R. Josa-Fombellida and J. P. Rincón-Zapatero, "Optimal asset allocation for aggregated defined benefit pension funds with stochastic interest rates," European Journal of Operational Research, vol. 201, no. 1, pp. 211-221, 2010.

[17] J. Gao, "Stochastic optimal control of DC pension funds," Insurance: Mathematics \& Economics, vol. 42, no. 3, pp. 11591164, 2008.

[18] G. Deelstra, M. Grasselli, and P.-F. Koehl, "Optimal investment strategies in a CIR framework," Journal of Applied Probability, vol. 37, no. 4, pp. 936-946, 2000.

[19] W. H. Fleming and T. Pang, "An application of stochastic control theory to financial economics," SIAM Journal on Control and Optimization, vol. 43, no. 2, pp. 502-531, 2004.

[20] C. Munk and C. Sørensen, "Optimal consumption and investment strategies with stochastic interest rates," Journal of Banking and Finance, vol. 28, no. 8, pp. 1987-2013, 2004.

[21] S. L. Heston, "A closed-form solution for options with stochastic volatility with applications to bond and currency options," Review of Financial Studies, vol. 6, no. 2, pp. 327-343, 1993.

[22] H. Kraft, "Optimal portfolios and Heston's stochastic volatility model: an explicit solution for power utility," Quantitative Finance, vol. 5, no. 3, pp. 303-313, 2005.

[23] W. H. Fleming and D. Hernández-Hernández, "An optimal consumption model with stochastic volatility," Finance and Stochastics, vol. 7, no. 2, pp. 245-262, 2003.

[24] G. Chacko and L. M. Viceira, "Dynamic consumption and portfolio choice with stochastic volatility in incomplete markets," Review of Financial Studies, vol. 18, no. 4, pp. 1369-1402, 2005. 
[25] Z. F. Li, Y. Zeng, and Y. Z. Lai, "Optimal time-consistent investment and reinsurance strategies for insurers under Heston's SV model, Insurance," Mathematics and Economics, vol. 50, no. 1, pp. 191-203, 2012.

[26] F. S. Sasha and T. Zariphopoulou, "Dynamic asset allocation and consumption choice in incomplete markets," Australian Economic Papers, vol. 44, no. 4, pp. 414-454, 2005.

[27] N. Castañeda-Leyva and D. Hernández-Hernández, “Optimal consumption-investment problems in incomplete markets with stochastic coefficients," SIAM Journal on Control and Optimization, vol. 44, no. 4, pp. 1322-1344, 2005.

[28] J. Liu, "Portfolio selection in stochastic environments," Review of Financial Studies, vol. 20, no. 1, pp. 1-39, 2007.

[29] D. Michelbrink and H. Le, "A martingale approach to optimal portfolios with jump-diffusions," SIAM Journal on Control and Optimization, vol. 50, no. 1, pp. 583-599, 2012.

[30] J. Li and R. Wu, "Optimal investment problem with stochastic interest rate and stochastic volatility: maximizing a power utility," Applied Stochastic Models in Business and Industry, vol. 25, no. 3, pp. 407-420, 2009.

[31] E.-J. Noh and J.-H. Kim, "An optimal portfolio model with stochastic volatility and stochastic interest rate," Journal of Mathematical Analysis and Applications, vol. 375, no. 2, pp. 510522, 2011. 


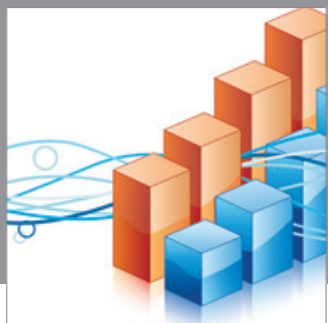

Advances in

Operations Research

mansans

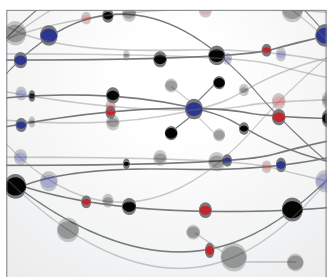

The Scientific World Journal
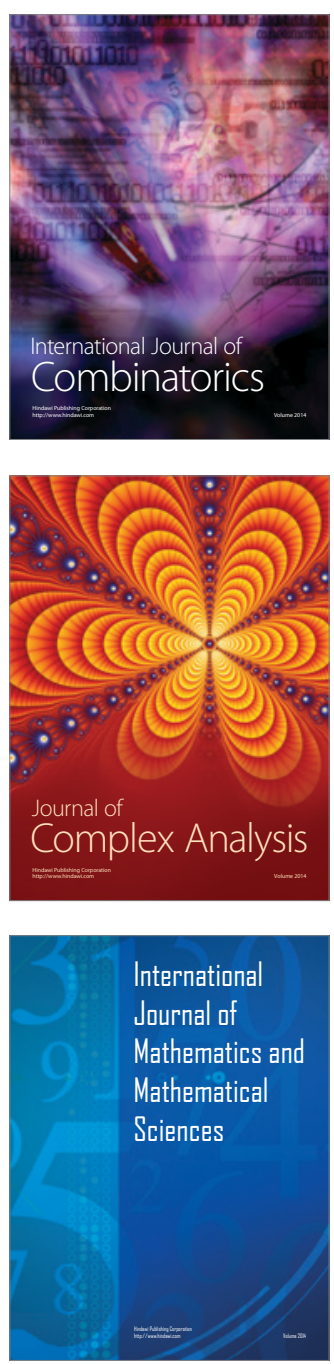
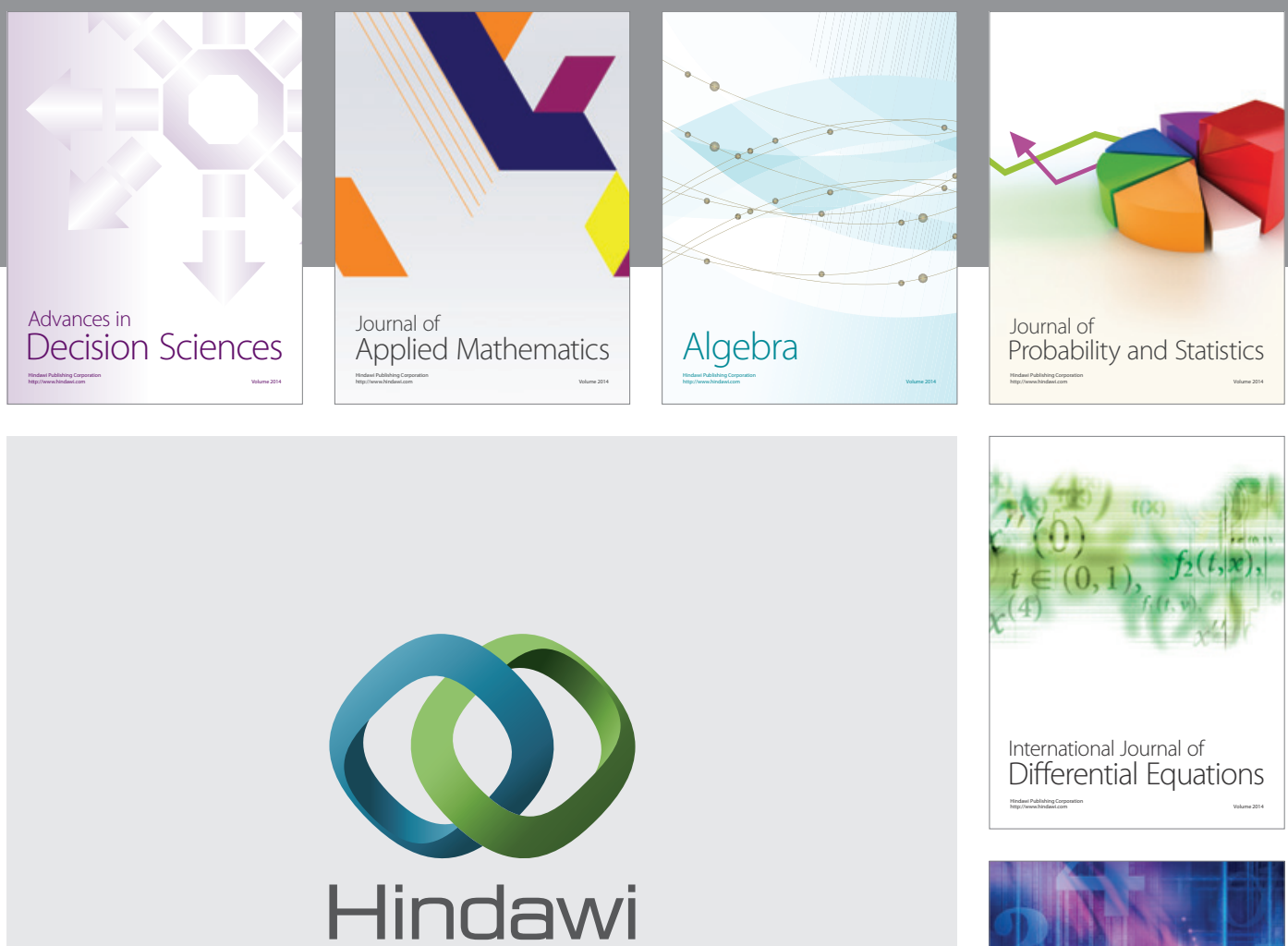

Submit your manuscripts at http://www.hindawi.com
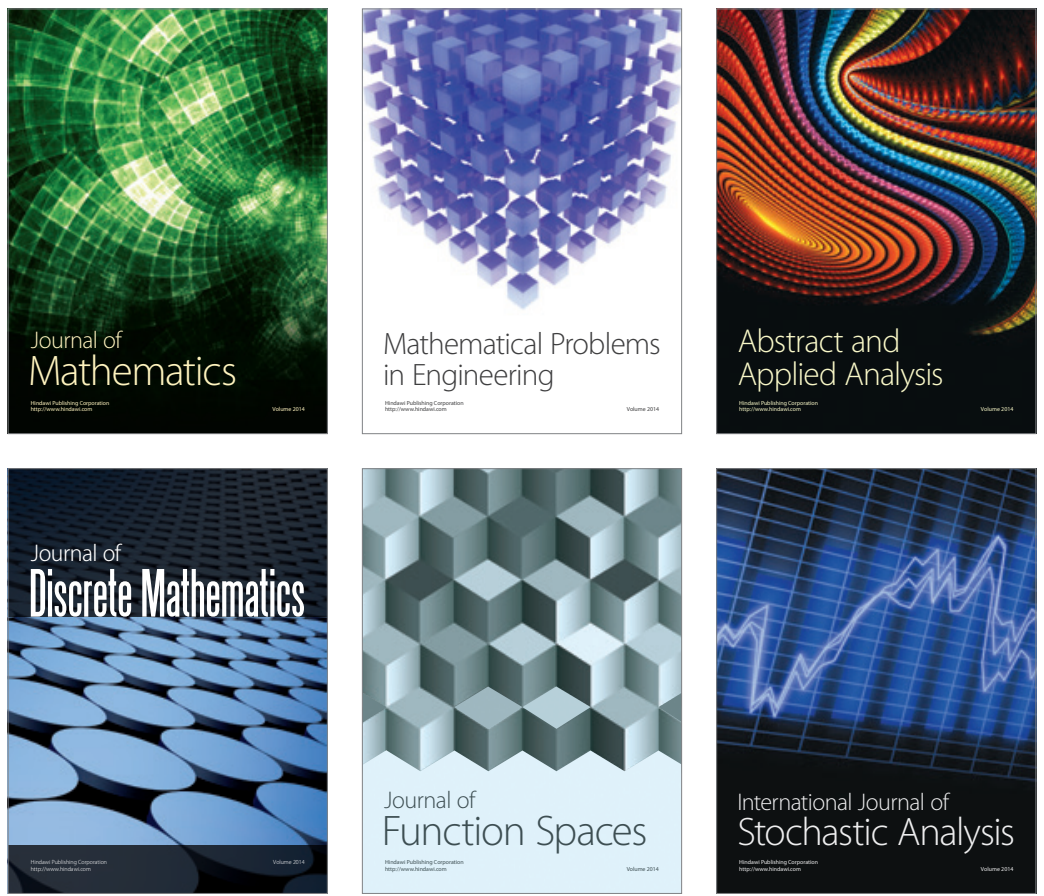

Journal of

Function Spaces

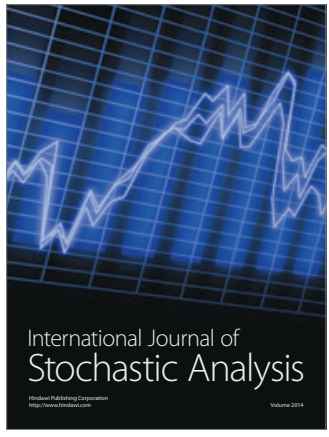

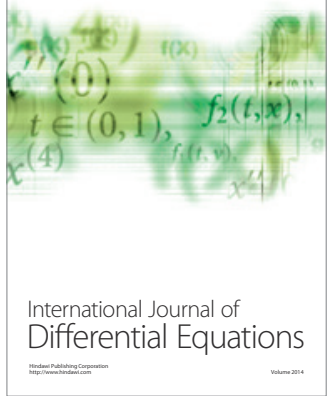
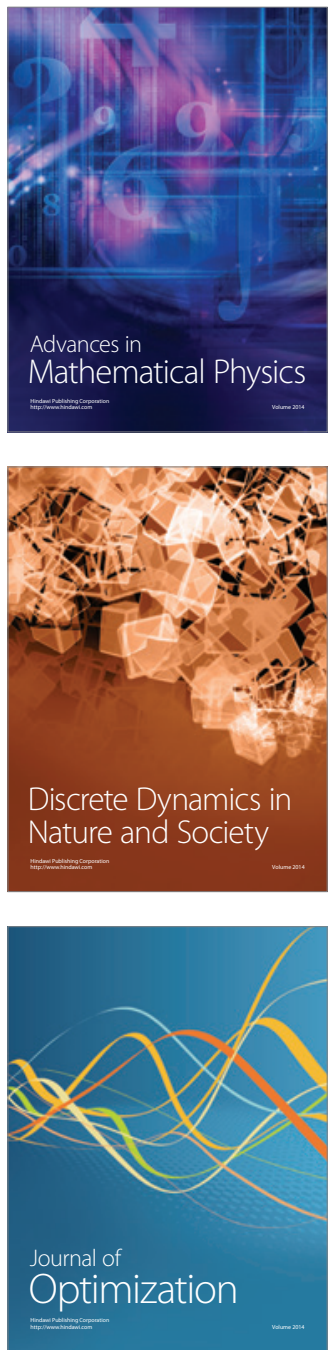